\title{
Increased expression of heat shock protein (HSP) 10 and HSP70 correlates with poor prognosis of nasopharyngeal carcinoma
}

This article was published in the following Dove Press journal: Cancer Management and Research

\author{
Juan Feng ${ }^{1,2}$ \\ Yuting Zhan' \\ Yuting Zhang' \\ Hongmei Zheng' \\ Weiyuan Wang ${ }^{3}$ \\ Songqing Fan'
}

'Department of Pathology, The Second Xiangya Hospital, Central South

University, Changsha, Hunan, People's

Republic of China; ${ }^{2}$ Clinical Laboratory of

Hunan Prevention and Treatment

Institute for Occupational Diseases,

Changsha, Hunan, People's Republic of

China; ${ }^{3}$ Department of Pathology,

Xiangya Hospital, Central South

University, Changsha, Hunan, People's

Republic of China
Correspondence: Songqing Fan Department of Pathology, The Second Xiangya Hospital, Central South University, 139 Renmin Middle Road,

Changsha, Hunan 4I00II, People's

Republic of China

Email songqingfan@csu.edu.cn
Background: Heat shock proteins (HSPs) are a large family of chaperones implicating in occurrence and progression of tumor. In our previous study, we found HSP10 correlates with poor prognosis of oral squamous cell carcinoma and astrocytoma. HSP70 is also an important part of this family and whether the alterations of HSP10 and HSP70 expression and their common expression correlates with carcinogenesis and progression of nasopharyngeal carcinoma (NPC) has not been reported.

Method: In this study, we investigate the correlation between the expression of HSP10 and HSP70 and clinicopathological characteristics in NPC by immunohistochemistry (IHC).

Results: Results indicated that positive expression of HSP10 and HSP70 was higher in NPC tissues (both $P<0.001$ ). Positive expression of HSP10 and HSP70 proteins, and common positive expression of the two HSPs analyzed in advanced clinical stages were higher than that in early clinical stages (All $P<0.05$ ). There was significantly higher expression of HSP10, HSP70, and common expression in NPC with LNM (lymph node metastasis) compared with NPC without LNM (All $P<0.05$ ). Interestingly, positive expression of HSP10 and HSP70 proteins and common expression had an evidently inverse correlation with survival status (All $P<0.05)$. Spearman's correlation analysis showed expression of HSP10 was positively associated with HSP70 $(\mathrm{r}=0.407, P<0 \quad 0.001)$. Kaplan-Meier analysis showed that the overall survival rates for NPC patients with positive expression of HSP10 and HSP70 and common expression were significantly lower than these patients with negative expression (All $P<0.05)$. Furthermore, positive expression of HSP10 and HSP70 proteins was identified as independent poor prognostic factors for NPC patients (both $P<0.05$ ) by Cox regression analysis.

Conclusion: In conclusion, HSP10 and HSP70 can serve as the poor prognostic factors for NPC patients.

Keywords: nasopharyngeal carcinoma, HSP10, HSP70, prognosis

\section{Introduction}

Nasopharyngeal carcinoma (NPC) is a malignant tumor originating from the pluripotent stem cells of nasopharyngeal epithelial tissue, which has a special geographic distribution and histopathology with the majority being undifferentiated non-keratinizing carcinomas and the incidence in Southern China and South-East Asia (endemic areas) being high. ${ }^{1,2}$ The etiology of NPC is thought to be associated with genetic susceptibility, Epstein-Barr virus (EBV) infection, dietary and environmental factors, the abnormal activation of signaling pathways and the aberrant expression of oncogenes/tumor-suppressor genes. ${ }^{3}$ The most common treatments 
for NPC include surgery, radiotherapy and chemotherapy or their combination. ${ }^{4}$ Although a lot of researches are conducted, no consensus has been reached on optimal treatment option for local recurrence and metastasis. Hence, further research on new targets of NPC is essential.

Heat shock proteins (HSPs) are one of the most evolutionarily conserved protein families whose expression is discovered to be strongly induced by heat shock and other physical and chemical stresses among many species. ${ }^{5}$ HSPs constitute a large family of proteins that are often classified based on their molecular weight: HSP10, HSP40, HSP60, HSP70, HSP 90, etc. ${ }^{6}$ Functionally, they work as molecular chaperones that assist the folding and refolding of other proteins and in the context of irreversibly or inaccurately misfolded proteins and promote their degradation. ${ }^{6}$ Researchers showed that HSPs also play important roles in many important biological or pathological processes including cell differentiation, gene expression, immune system regulation, cellular senescence, programmed cell death, and carcinogenesis. ${ }^{7-12}$ HSPs are involved in carcinogenesis, angiogenesis, cell proliferation and invasiveness. ${ }^{12-14}$ HSPs are proved to implicate in the occurrence and progression of tumor. ${ }^{15}$ Many biological or pathological processes have been confirmed to relate to HSPs such as immunoregulation, apoptosis, drug resistance of tumors, and HSPs also participate in oncogene activation and tumor suppressor gene inactivation. ${ }^{16-21}$ Aberrant expression of the HSPs has been detected in various tumors, including colorectal, breast, prostate, lung, ovarian, gastric, oral and esophageal cancer. ${ }^{2,22}$ Our previous study has demonstrated that elevated expression of HSP10 protein inhibits apoptosis and associates with poor prognosis of astrocytoma, high expression of heat shock protein 10 correlates negatively with estrogen/progesterone receptor status and predicts poor prognosis in invasive ductal breast carcinoma, and high expression of HSP10 is associated with poor prognosis in oral squamous cell carcinoma. ${ }^{23-25} \mathrm{We}$ also found that high expression of HSP90 could be used as a novel independent poor prognostic biomarker for patients with NPC. ${ }^{26}$ So far, the correlation between expression of HSP10 and HSP70 proteins and clinicopathological characteristics of NPC has not been reported.

In the present study, expression of HSP10 and HSP70 proteins in 445 cases of NPC and 54 cases of non-cancerous nasopharyngeal control tissue was detected by immunohistochemistry (IHC). Also, we analyzed the correlations between the expression of HSP10 and HSP70 proteins and clinicopathological characteristics and prognostic implications in NPC.

\section{Materials and methods}

\section{Ethics statement}

All research protocols were approved by the Ethics Review Committee of the Second Xiangya Hospital of Central South University (Scientific and Research Ethics Committee, No. Y202/2014). And samples were obtained with informed consent. If the patient is a minor child, a written consent would be signed by a close relative, caretaker or guardian on behalf of the minor/child participant who participated in our study.

\section{Tissue samples and clinical data}

In this study, 445 cases of NPC with their age ranging from 14 to 81 years (median, 47.4 years), and 54 cases of non-cancerous nasopharyngeal control epithelium were randomly obtained from the department of pathology at The Second Xiangya Hospital of Central South University (Changsha, China). Patients had not been previously treated with radiotherapy or chemotherapy at the time of original biopsy. Complete clinical record and follow-up data were available for all patients. All 445 cases of NPC patients were continued to follow-up for 10 years (during the period from 2000 to 2010). All patients had a defined histological diagnosis of NPC according to WHO histological classification of the NPC. Based on the criteria of the 8th edition of the AJCC/UICC TNM staging system of NPC (2017), these patients had been divided into clear staging classification of the current study.

There were 445 cases of NPC, including 29 cases of differentiated non-keratinizing NPC and 416 cases of undifferentiated non-keratinizing NPC. Clinical stages of NPC were partitioned as follows: 4 cases of clinical stage I, 101 cases of stage II, 196 cases of stage III, and 144 cases of stage IV, which included 359 cases of NPC with lymph node metastasis and 86 cases of NPC without lymph node metastasis (LNM). Every patient received Epstein-Barr virus (EBV) antibody detection via ELISA clinically, and EB virus-encoded RNAs (EBER) were investigated in paraffin sections of NPC by in situ hybridization. The results showed that $99 \%$ of NPC patients were EBV antibodypositive and 95\% NPC specimens were positive for EBV. Overall survival (OS) time was calculated from the time of diagnosis to the date of death or last contact. A total of 345 patients $(77.5 \%)$ were alive with a mean follow-up period of 32.9 months (2-120 months). 


\section{IHC and semi-quantitative scores}

In our previous study, we found HSP10 correlates with poor prognosis of oral squamous cell carcinoma and astrocytoma. ${ }^{23-25}$ HSP70 is also an important part of this family and whether the alterations of HSP10 and HSP70 expression and their common expression correlates with carcinogenesis and progression of NPC has not been reported. So, in this study, we investigate the correlation between the expression of HSP10 and HSP70 proteins and clinicopathological characteristics in NPC by IHC. The IHC staining for HSP10 and HSP70 in NPC samples was carried out using ready-to-use Envision $\mathrm{TM}^{+}$Dual Link System-HRP methods (Dako, Carpinteria, CA, USA). According to our laboratory experience, ${ }^{23-26}$ the staining conditions for each antibody were adjusted. In brief, each section was deparaffinized and rehydrated, and high-temperature antigen retrieval was achieved for all antibodies by heating the samples in $0.01 \mathrm{M}$ citrate buffer in a domestic microwave oven at full power $(750 \mathrm{~W})$ for 15 mins. Methanol containing 3\% $\mathrm{H}_{2} \mathrm{O}_{2}$ was applied for 15 mins to inactivate endogenous peroxidase. The slides were incubated with a rabbit polyclonal antibody against human, a 1:2500 dilution of the primary antibody to HSP10 (Mouse monoclonal antibody, Catalog: sc376313, Santa Cruz Biotechnology, Inc., Dallas, TX, USA), a 1:1000 dilution of the primary antibody to HSP70 (Rabbit monoclonal antibody, Catalog: 4873; Cell Signaling, Danvers, MA, USA) at $4^{\circ} \mathrm{C}$ overnight. Slides were rinsed with PBS three times for 5 mins each. After the incubation with secondary antibody, the visualization signal was conducted with 3,3'-diaminobenzidine tetrachloride. Subsequently, all slides were counterstained with hematoxylin. Positive control slides were included in every experiment in addition to the internal positive control. At the same time, the specificity of the antibody was determined with matched $\operatorname{IgG}$ isotype antibody as a negative control.

Immunohistochemical staining was evaluated independently by SF and JF who were blinded to the clinicopathological data, at 200× magnification light microscopy. HSP10 and HSP70 proteins were located in the cytoplasm. A semi-quantitative evaluation of HSP10 and HSP70 expression was performed using a method described in the literature ${ }^{26-28}$ : staining intensity for HSP10 and HSP70 were divided into four grades (intensity scores): as 0 (negative, no staining), 1 (weak, light brown), 2 (moderate, brown), and 3 (strong, dark brown). The positive percentage was divided into five grades (percentage scores): 0 (0\%), 1 (1-25\%), 2 (26-50\%), 3 (51-75\%), and $4(76-100 \%)$. Staining positivity was determined by the formula: overall scores $=$ percentage score $\times$ intensity score. The result of the staining scores was used as the final staining score for HSP10 and HSP70 (0-12). Optimal cut off score was 4, which was on the basis of the OS of NPC patients using the log-rank test. A score of $\geq 4$ was considered a high (positive) expression of HSP10 and HSP70 while scores $<4$ were considered low (negative) expression of HSP10 and HSP70. Agreement between the two evaluators was $97 \%$, and all assess differences were solved through discussion between the two evaluators.

\section{Statistical analyses}

SPSS18.0 statistical software (SPSS Inc., Chicago, IL, USA) was used to test the correlation between the expression of HSP10 and HSP70 proteins and their clinicopathological characteristics in NPC by chi-square test. The Spearman's rank correlation coefficient was used to assess the significance of the association among the expression of HSP10 and HSP70 proteins in NPC. Kaplan-Meier survival analysis was performed for OS curves and statistical significance was assessed using the log-rank test. To identify whether expression of HSP10 and HSP70 proteins was the independent prognostic factors of OS for NPC, multivariate analysis using the Cox proportional hazard regression model was performed. All $P$-values were based on the two-sided statistical analysis and $P<0.05$ was considered to be statistically significant.

\section{Results}

\section{Association between expression of HSPIO and HSP70 proteins and clinicopathological characteristics of NPC}

We examined the positive expression and cellular location of HSP10 and HSP70 in NPC and non-cancerous control nasopharyngeal epithelium by IHC. Strong positive granule expression of HSP10 protein was distributed in the cytoplasm of the NPC (Figure 1A), weak expression of HSP10 protein was shown in the control nasopharyngeal epithelial cells (Figure 1B), and there was negative staining of HSP10 in the control NPC (Figure 1C). Strong expression of HSP70 protein was located in the cytoplasm and nuclear of NPC (Figure 1D), moderated positive staining of HSP70 was indicated in the cytoplasm and nuclear of nasopharyngeal epithelial cells (Figure 1E), and there 


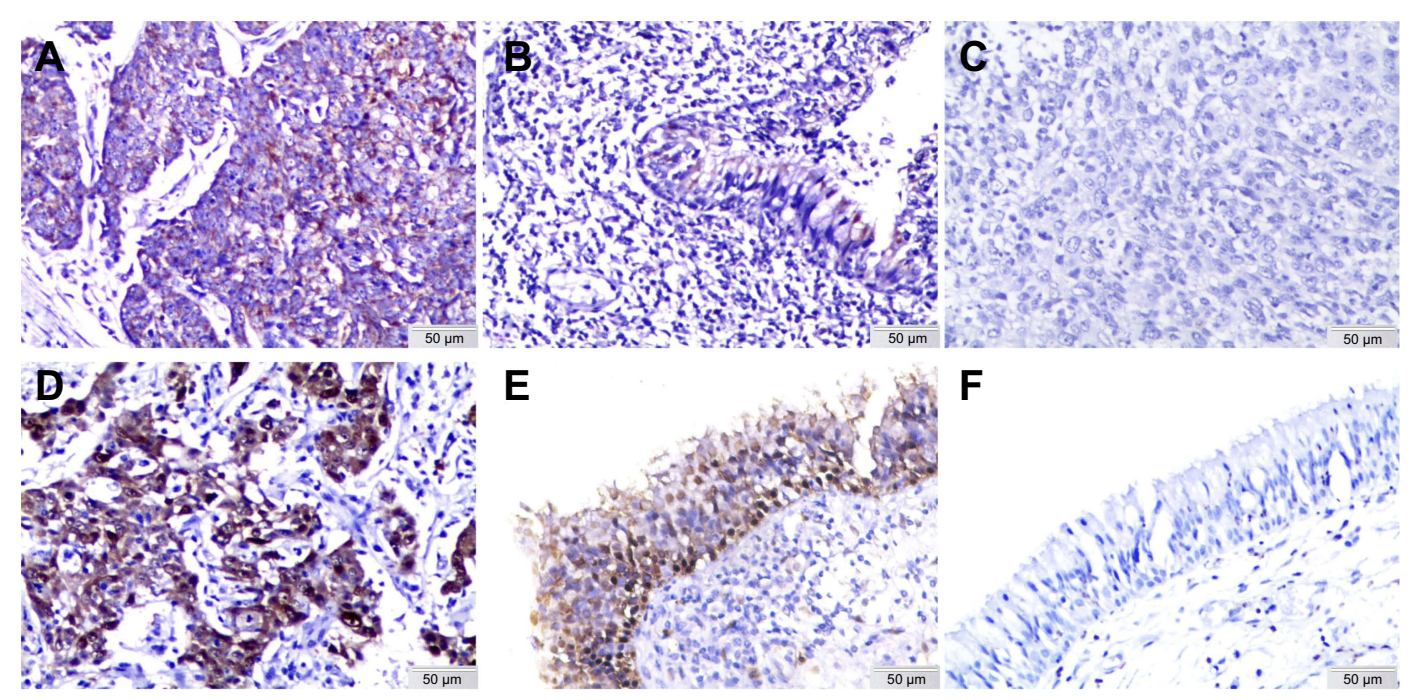

Figure I Expression of HSPIO and HSP70 in NPC and non-cancerous control nasopharyngeal epithelium was detected by IHC. The expression of HSPIO and HSP70 was detected by IHC using specific antibodies as described in "Materials and methods" section. Strong positive granule expression of HSPIO protein was distributed in the cytoplasm of the NPC (A), weak expression of HSPIO protein was showed in the control nasopharyngeal epithelial cells (B), and there was negative staining of HSPIO in the NPC (C). Strong expression of HSP70 protein was located in the cytoplasm and nucleus of NPC (D), moderated positive staining of HSP70 was indicated in the cytoplasm and nucleus of nasopharyngeal epithelial cells (E), and there was no expression of HSP70 protein in the control nasopharyngeal epithelial cells (F) (IHC, 3,3'diaminobenzidine staining, original magnification $\times 200$ ).

Abbreviations: HSP, heat shock protein; NPC, nasopharyngeal carcinoma; IHC, immunohistochemistry.

was no expression of HSP70 protein in the control nasopharyngeal epithelial cells (Figure 1F).

Expression level of HSP10 and HSP70 proteins in 445 cases of NPC and 54 cases of non-cancerous control nasopharyngeal epithelium was presented in bar graph (Figure 2). In the 445 cases of NPC, positive percentage of HSP10 and HSP70 expression was 75.7\% (337/445) and $67.9 \%(302 / 445)$. In the 54 cases of non-cancerous control nasopharyngeal epithelium, positive percentage of HSP10 and HSP70 expression was $16.7 \%(9 / 54)$ and $38.9 \%$ (21/54). The positive percentage of HSP10 and HSP70 expression was significantly higher in the NPC than that in the non-cancerous control nasopharyngeal epithelium $(P<0.001$ and $P<0.001$, respectively).

The associations between expression of HSP10 and HSP70 proteins and clinicopathological characteristics of NPC including age, gender, histological type, clinical stages, lymph node metastasis and survival status were investigated through univariate chi-square test, and results are summarized in Table 1. Positive percentage of HSP 10 and HSP70 expression in advanced clinical stages were significantly higher than that in early clinical stages ( $P=0.003$ and $P=0.007$, respectively). Moreover, common positive expression of two proteins above in NPC with advanced clinical stages was significantly higher than that in early clinical stages $(P=0.030)$. A comparison of NPC with lymph node metastasis and without lymph node

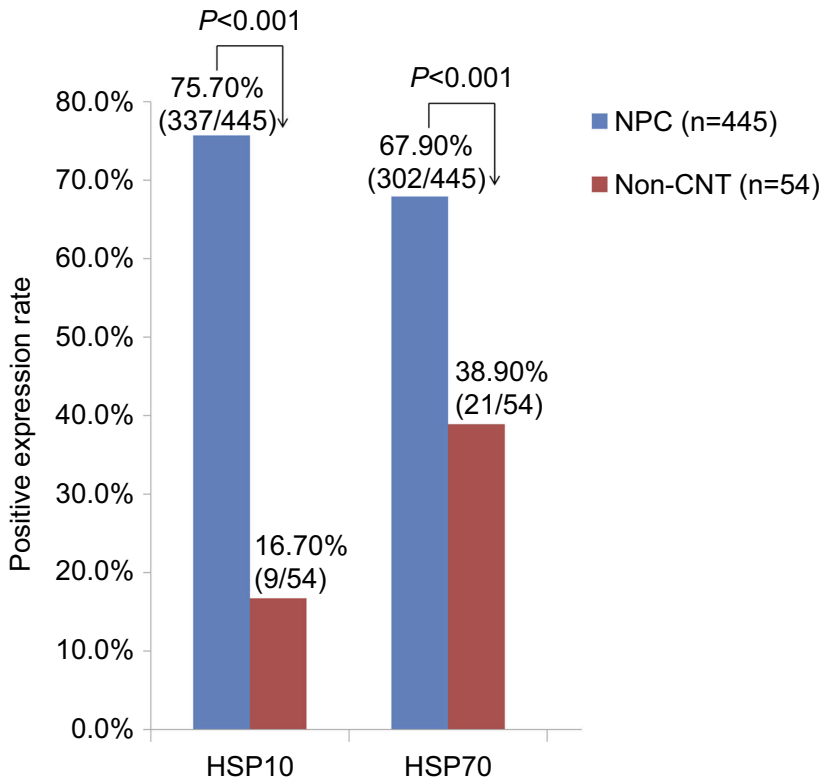

Figure 2 Bar graph showed that the expression levels of HSPIO and HSP70 proteins in NPC and non-cancerous nasopharyngeal control epithelium. In the 445 cases of NPC, positive percentage of HSPIO and HSP70 expression was $75.7 \%(337 / 445)$ and $67.9 \%(302 / 445)$. In the 54 cases of non-cancerous control nasopharyngeal epithelium, positive percentage of HSPIO and HSP70 was $16.7 \%$ (9/ $45)$ and $38.9 \%(21 / 45)$. There were significantly higher positive expression of HSPIO and HSP70 proteins in NPC compared to the non-cancerous control nasopharyngeal epithelium $(P<0.00 \mathrm{I}$ and $P<0.00 \mathrm{I}$, respectively).

Abbreviations: HSP, heat shock protein; NPC, nasopharyngeal carcinoma.

metastasis showed that there was evidently higher positive expression of HSP10 and HSP70 proteins $(P=0.001$ and $P=0.003$, respectively) in NPC with lymph node 
Table I Association between expression of HSPI0 and HSP70 proteins and clinicopathological characteristics of NPC ( $\mathrm{n}=445$ )

\begin{tabular}{|c|c|c|c|c|c|c|c|c|c|}
\hline \multirow[t]{2}{*}{ Characteristics (n) } & \multicolumn{3}{|l|}{ HSPIO } & \multicolumn{3}{|l|}{ HSP70 } & \multicolumn{3}{|c|}{$H S P I O / H S P 70^{a}$} \\
\hline & $\mathbf{P}(\%)$ & $\mathbf{N}(\%)$ & $P$-value & $\mathbf{P}(\%)$ & $\mathbf{N}(\%)$ & $P$-value & $\mathbf{P}(\%)$ & $\mathbf{N}(\%)$ & $P$-value \\
\hline \multicolumn{10}{|l|}{ Age (year) } \\
\hline$<50(n=259)$ & $193(74.5)$ & $66(25.5)$ & $0.48 \mathrm{I}$ & $181(69.9)$ & $78(30.1)$ & 0.282 & $158(61.0)$ & $101(39.0)$ & 0.461 \\
\hline$\geq 50(n=186)$ & I 44 (77.4) & $42(22.6)$ & & $12 \mid(65.1)$ & $65(34.9)$ & & $107(57.5)$ & $79(42.5)$ & \\
\hline \multicolumn{10}{|l|}{ Gender } \\
\hline Female $(n=114)$ & $84(73.7)$ & $30(26.3)$ & 0.555 & $79(69.3)$ & $35(30.7)$ & 0.704 & $68(59.6)$ & $46(40.4)$ & 0.980 \\
\hline Male $(n=33 I)$ & $253(76.4)$ & $78(23.6)$ & & $223(67.4)$ & $108(32.6)$ & & 197(59.5) & $134(40.5)$ & \\
\hline \multicolumn{10}{|l|}{ Histological type } \\
\hline DNC (n=29) & $21(72.4)$ & $8(27.6)$ & 0.667 & $20(69.0)$ & $9(31.0)$ & 0.896 & $17(58.6)$ & $12(41.4)$ & 0.916 \\
\hline UDNC $(n=416)$ & $316(76.0)$ & $100(24.0)$ & & $282(67.8)$ & $134(32.2)$ & & $248(59.6)$ & $168(40.4)$ & \\
\hline \multicolumn{10}{|l|}{ Clinical stages } \\
\hline Stages I and II $(n=105)$ & $68(64.8)$ & $37(35.2)$ & $0.003^{*}$ & $60(57.1)$ & $45(42.9)$ & $0.007 *$ & $53(50.5)$ & $52(49.5)$ & $0.030^{*}$ \\
\hline Stages III and IV $(n=340)$ & $269(79.1)$ & 71 (20.9) & & $242(7 \mid .2)$ & $98(28.8)$ & & $212(62.4)$ & $128(37.6)$ & \\
\hline \multicolumn{10}{|l|}{ LNM status } \\
\hline LNM (n=359) & 284 (79.1) & $75(20.9)$ & $0.001 *$ & $255(71.0)$ & $104(29.0)$ & $0.003 *$ & $222(61.8)$ & 137 (38.2) & $0.045^{*}$ \\
\hline No LNM $(n=86)$ & $53(6 \mid .6)$ & $33(38.4)$ & & 47 (54.7) & $39(45.3)$ & & $43(50.0)$ & $43(50.0)$ & \\
\hline \multicolumn{10}{|l|}{ Survival status } \\
\hline Alive $(n=345)$ & $250(72.5)$ & $95(27.5)$ & $0.003^{*}$ & $221(64.1)$ & $124(35.9)$ & $0.001 *$ & $190(55.1)$ & I55 (44.9) & $0.000 * *$ \\
\hline Dead $(n=100)$ & $87(87.0)$ & $13(13.0)$ & & 81 (8I.0) & $19(19.0)$ & & $75(75.0)$ & $25(25.0)$ & \\
\hline
\end{tabular}

Notes: The data were tested using the two-tailed chi-square test, where the $P$-value below 0.05 were considered significant. $* P<0.05$, $* * P<0.00 \mathrm{I}$. ${ }^{\mathrm{P}} \mathrm{P}$ is common positive expression of HSPIO and HSP70, and $\mathrm{N}$ is any one negative expression of HSPIO and HSP70.

Abbreviations: DNC, Differentiated non-keratinized nasopharyngeal carcinoma; UDNC, Undifferentiated non-keratinized nasopharyngeal carcinoma; LNM, lymph node metastasis; P, positive; N, negative.

metastasis. NPC patients with lymph node metastasis showed importantly higher common positive expression of two proteins above than NPC patients without lymph node metastasis $(P=0.045)$. Interestingly, we found that NPC patients in the dead group had a significantly higher positive expression of HSP10 and HSP70 proteins than that in the alive group $(P=0.003$ and $P=0.001$, respectively). A conjoint analysis indicated that common positive expression of HSP10 and HSP70 had an evidently inverse correlation with survival status of NPC patients $(P<0.001)$.

\section{The pairwise correlation between} expression of HSPIO and HSP70 in NPC Moreover, the association between positive expression of HSP10 and HSP70 proteins in 445 cases of NPC was analyzed by Spearman's rank correlation analysis. Among 445 cases of NPC cases, 265 patients were found to express HSP10 and HSP70 commonly, 72 patients with positive expression of HSP10 protein and negative expression of HSP70 protein, 37 patients with negative expression of HSP10 protein and positive expression of HSP70 protein, and 71 patients with both negative expression of HSP10 and HSP70 proteins. Analysis showed that positive expression of HSP10 was significantly associated with HSP70 protein by Spearman's rank correlation test $(\mathrm{r}=0.407, P<0.001)$.

\section{Expression of HSPIO and HSP70 is positively related to the poor overall survival rates of NPC patients}

Since positive expression of HSP10 and HSP70 proteins was associated with the survival status of NPC patients by the chi-square tests, we further evaluated the relationship between positive expression of HSP10 and HSP70 proteins and the OS of NPC patients through Kaplan-Meier survival curve analysis with log-rank significance test. Figure 3 illustrates the Kaplan-Meier survival plots for NPC patients with expression of HSP10 (Figure 3A), HSP70 (Figure 3B) and common positive expression of two proteins above (Figure $3 \mathrm{C}$ ). The OS rates for NPC patients with positive expression of HSP10 and HSP70 and common positive expression of two proteins above 
A

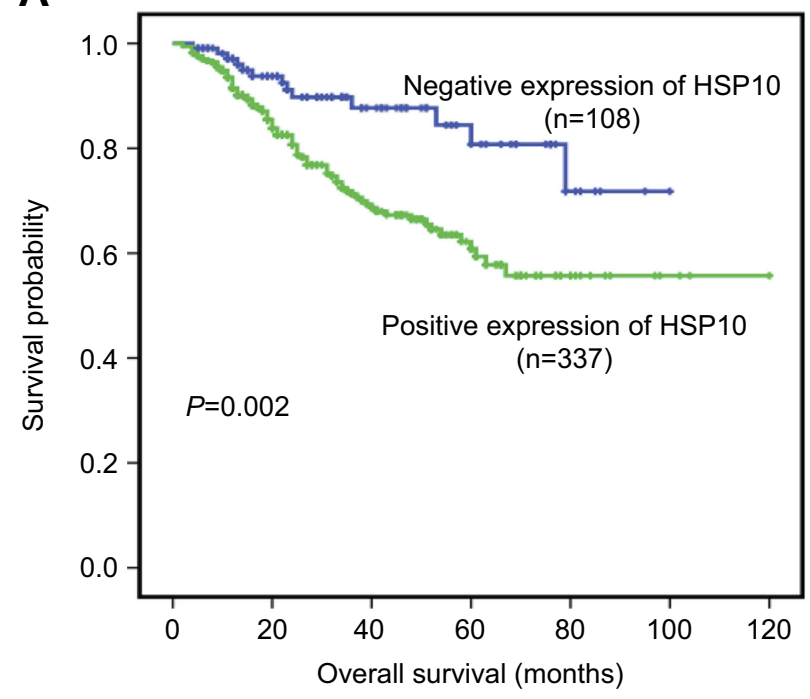

B

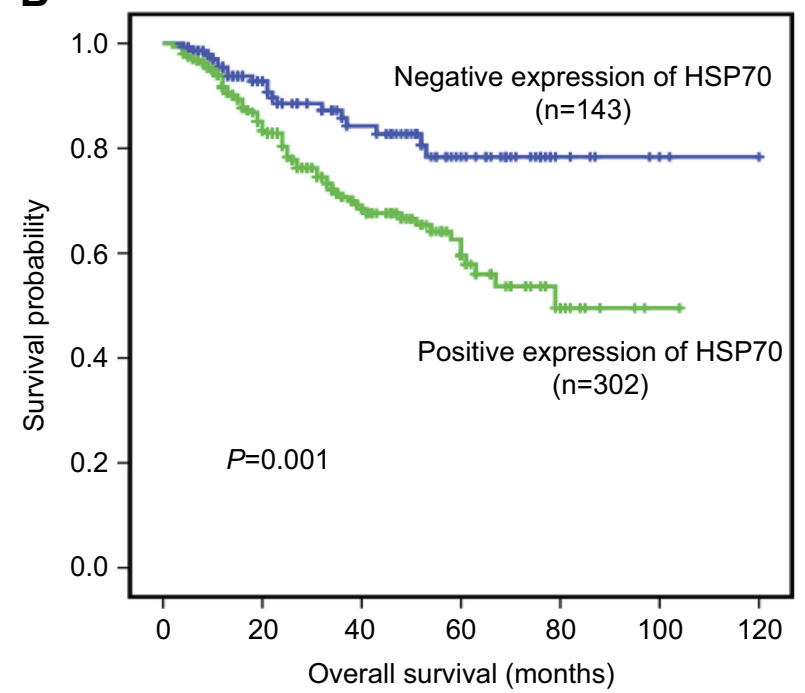

\section{C}

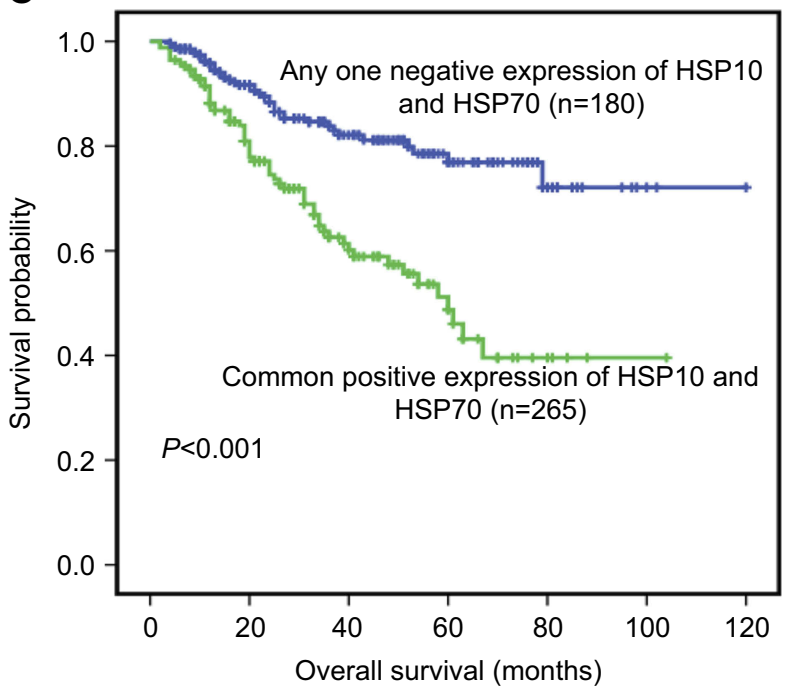

Figure 3 Kaplan-Meier overall survival curves of NPC patients with expression of HSPIO and HSP70 and common positive expression of two proteins above. Kaplan-Meier analysis to plot the survival curve of 445 cases of NPC patients with expression of HSPIO and HSP70 proteins and statistical significance were assessed using the log-rank test. (A) Kaplan-Meier curves showed worse overall survival rates for NPC patients with positive expression of HSPI0 compared to patients with HSPI0 negative staining $(P=0.002$, two sided). (B) Kaplan-Meier curves showed worse overall survival rates for NPC patients with positive expression of HSP70 compared to patients with HSP70 negative staining $(P=0.001$, two sided). (C) Kaplan-Meier curves showed worse overall survival rates for NPC patients with common positive expression of HSPIO and HSP70 proteins compared to patients with any one negative staining of two proteins above $(P<0.00 \mathrm{I}$, two sided).

Abbreviations: HSP, heat shock protein; NPC, nasopharyngeal carcinoma.

were significantly lower than these patients with negative expression $(P=0.002, P=0.001$ and $P<0.001$, respectively).

In addition to univariate analysis, multivariate Cox proportional hazard regression analysis was also carried out to further investigate whether the expression of HSP10 and HSP70 proteins is the independent prognostic factors of patients with NPC in this study. In multivariate analysis, factors including gender, age, histological type, clinical stages, lymph node metastasis status, treatment strategy and positive expression of HSP10 and HSP70 proteins were considered. Results revealed that positive expression of HSP10 and HSP70 proteins can be used as independent poor prognostic factors for NPC patients $(P=0.018$ and $P=0.026$, respectively), as well as clinical stages and lymph node metastasis status $(P=0.024, P=0.019$, respectively) (Table 2).

\section{Discussion}

HSPs are molecular chaperones which are involved in the cellular processes of protein assembly, folding and 
Table 2 Summary of multivariate analysis of Cox proportional hazard regression for overall survival in 445 cases of NPC patients

\begin{tabular}{|l|l|l|l|l|l|l|l|}
\hline Variables & B & SE & Wald & Sig & Exp (B) & \multicolumn{2}{|c|}{ 95.0\% Cl for Exp (B) } \\
\cline { 3 - 7 } & & & & & & Lower & Upper \\
\hline Age & 0.376 & 0.208 & 3.262 & 0.071 & 1.457 & 0.968 & 2.191 \\
Gender & -0.603 & 0.273 & 1.867 & 0.175 & 0.347 & 0.220 & 1.934 \\
Histological type & -0.089 & 0.467 & 0.037 & 0.848 & 0.914 & 0.366 & 2.285 \\
Clinical stages & 0.668 & 0.296 & 5.088 & $0.024 *$ & 1.950 & 1.091 & 3.484 \\
LNM status & 0.935 & 0.398 & 5.526 & $0.019 *$ & 2.546 & 1.168 & 5.550 \\
Treatment strategy & -0.332 & 0.208 & 2.551 & 0.110 & 0.718 & 0.477 & 1.078 \\
HSPIO & 1.440 & 0.608 & 5.600 & $0.018^{*}$ & 4.220 & 1.281 & 13.907 \\
HSP70 & 1.265 & 0.567 & 4.968 & $0.026 *$ & 3.542 & 1.165 & 10.773 \\
\hline
\end{tabular}

Note: Multivariate analysis of Cox proportional hazard regression, $* P<0.05$.

Abbreviations: LNM, lymph node metastasis; B, beta coefficient (B); SE, standard error; Exp (B), exponentiation of the B coefficient.

refolding, translocation and degradation, and can help to maintain and ensure the conformation and functional stability of various proteins in the cell under stress conditions. ${ }^{27-29}$ Molecular chaperones have various functions and are of great importance to the cell. In certain case, the chaperone can be both structurally and functionally normal but just get participated in pathways that lead to disease. For instance, the post-translational modification of the chaperone could alter its subcellular localization and subsequent function. ${ }^{17-20,28-30}$ Moreover, high expression of chaperone can lead to superabundant protein synthesis, which is a common feature of a wide variety of tumors.

HSPs constitute a large extended family of proteins and the role of HSPs in tumor cells has been widely studied in recent years. HSPs are useful biomarkers for carcinogenesis and the aggressiveness of certain cancers. ${ }^{29-31}$ It was reported that overexpression of HSP10 in adenocarcinoma compared to healthy mucosa of large bowel, and interestingly, HSP10 was found in both the epithelium and the lamina propria. ${ }^{21,32}$ Moreover, HSP10 was also confirmed to correlate with lymph node metastases in large bowel carcinoma. ${ }^{33}$ In terms of mechanism, HSP10 and HSP60 could increase the abundance of the anti-apoptotic Bcl-xl and $\mathrm{Bcl}-2$, and reduced the protein content of the proapoptotic Bax in the study of doxorubicin in cardiac muscle cells. ${ }^{34}$ Apart from HSP10, HSP70 was also proved to highly express in many kinds of malignant tumor and might act as a marker for poor prognosis, and targeting HSP70 could be a possible therapy for cancer treatment. ${ }^{35}$ For example, HSP70 level was significantly elevated in human pancreatic cancer tissue than normal pancreatic tissue at the tumor margins, and deeper study demonstrated that HSP70 opposed apoptosis in transformed cells at multiple levels. ${ }^{36}$ It was also reported that HSP70 expression levels were higher in hepatocellular carcinoma than those of liver cirrhosis, chronic hepatitis, and controls and HSP70 could be potential biomarkers for HCC diagnosis, ${ }^{37}$ so as in patients with gastric carcinoma, especially of intestinal type ${ }^{38}$ However, in blood test, it is controversial for HSP70 expression. Gunaldi's group found that serum levels of HSP70 in patients with CRC were significantly higher compared with the control group, whereas Tang et al believed that decreased circulating HSP70, in combination with elevated CEA and CA 19-9, could be utilized in the diagnosis of early (stages I and II) lung cancer. ${ }^{39,40}$

Expression of HSPs is detectable and associated with poor prognosis in a variety of human cancers, including breast cancer, melanoma and clear cell renal cell carcinoma. ${ }^{24,41-43}$ In our present study, results showed that positive expression of HSP10 and HSP70 proteins was significantly increased in NPC compared with noncancerous control nasopharyngeal epithelial tissue. The expression of HSP10 and HSP70 proteins in NPC patients with lymph node metastasis was also higher than that in NPC patients without lymph node metastasis. What is noteworthy is that those NPC patients with common positive expression of two proteins above might have a lower survival probability than NPC patients with any one negative staining of three proteins. Thus, it suggested that HSP10 and HSP70 might play important roles in promoting the development and progression of NPC. Furthermore, expression of HSP10 and HSP70 proteins was positively related to each other. These findings indicated that increased expression of HSPs could play an important part in the pathogenesis of NPC. We also showed that the NPC patients with positive expression of HSP10 and HSP70 proteins had a notably shorter survival 
time than these patients with negative staining of HSP10 and HSP70. Moreover, multivariate analysis proved that positive expression of HSP10 and HSP70 proteins was independent factors of poor prognosis in NPC regardless of clinical stages and state of lymph node metastasis. Our results were consistent with previous study, ${ }^{24,41-43}$ and implicate the important roles that HSP family might play in cancer occurrence and progression. Therefore, aberrant expression of HSP10 and HSP70 proteins might be employed as novel prognostic markers for NPC.

In summary, this is the first report about the positive relationship between high expression of HSP10 and HSP70 proteins and the poor OS rates in NPC. Furthermore, positive expression of HSP10 and HSP70 proteins could serve as independent prognostic factors for predicting a poor prognosis in patients with NPC.

\section{Acknowledgments}

The work was supported by the National Natural Science Foundation of China (Nos. 81472773, 81773218, 81703009,81802791 and 81272566) and the Natural Science Foundation of Hunan Province (Nos. 2018JJ3858 and 2017JJ3457).

\section{Disclosure}

The authors report no conflicts of interest in this work.

\section{References}

1. Huang WB, Chan JYW, Liu DL. Human papillomavirus and World Health Organizationtype III nasopharyngeal carcinoma: Multicenter study from an endemic area in Southern China. Cancer. 2018;124 (3):530-536.

2. El-Naggar AK, Chan JKC, Takata T, Grandis JR, Slootweg PJ. The fourth edition of the head and neck World Health Organization blue book: editors' perspectives. Hum Pathol. 2017;66:10-12. doi:10.10 16/j.humpath.2017.05.014

3. Chou J, Lin YC, Kim J, et al. Nasopharyngeal carcinoma-review of the molecular mechanisms of tumorigenesis. Head Neck. 2008;30(7):946963. doi: $10.1002 /$ hed.20833

4. Stoker SD, van Diessen JN, de Boer JP, et al. Current treatment options for local residual nasopharyngeal carcinoma. Curr Treat Options Oncol. 2013;14(4):475-491. doi:10.1007/s11864-013-0261-5

5. Georgopoulos C, Welch WJ. Role of the major heat shock proteins as molecular chaperones. Annu Rev Cell Biol. 1993;9:601-634. doi:10.1146/annurev.cb.09.110193.003125

6. Li Z, Srivastava P. Heat-shock proteins. Curr Protoc Immunol. 2004; Appendix 1:Appendix 1T. doi: 10.1002/0471142735.ima01ts58

7. Walsh D, Grantham J, Zhu XO, et al. The role of heat shock proteins in mammalian differentiation and development. Environ Med. 1999;43 (2):79-87.

8. Voellmy R. Transduction of the stress signal and mechanisms of transcriptional regulation of heat shock/stress protein gene expression in higher eukaryotes. Crit Rev Eukaryot Gene Expr. 1994;4 (4):357-401.
9. Pockley AG, Muthana M, Calderwood SK. The dual immunoregulatory roles of stress proteins. Trends Biochem Sci. 2008;33(2):71-79. doi:10.1016/j.tibs.2007.10.005

10. Manerba M, Di Ianni L, Govoni M, Roberti M, Recanatini M, Di Stefano G. LDH inhibition impacts on heat shock response and induces senescence of hepatocellular carcinoma cells. Eur J Pharm Sci. 2017;105:91-98. doi:10.1016/j.ejps.2017.05.015

11. Shin MK, Jeong KH, Choi H, Ahn H-J, Lee M-H. Heat shock protein 90 inhibitor enhances apoptosis by inhibiting the AKT pathway in thermalstimulated SK-MEL-2 human melanoma cell line. J Dermatol Sci. 2018;90(3):357-360. doi:10.1016/j.jdermsci.2018.02.004

12. Czarnecka AM, Campanella C, Zummo G, Cappello F. Mitochondrial chaperones in cancer: from molecular biology to clinical diagnostics. Cancer Biol Ther. 2006;5(7):714-720. doi:10.4161/cbt.5.7.2975

13. Sanderson S, Valenti M, Gowan S, et al. Benzoquinone ansamycin heat shock protein 90 inhibitors modulate multiple functions required for tumor angiogenesis. Mol Cancer Ther. 2006;5(3):522-532. doi:10.1158/1535-7163.MCT-05-0439

14. Zhao L, Liu L, Wang S, et al. Differential proteomic analysis of human colorectal carcinoma cell lines metastasis-associated proteins. J Cancer Res Clin Oncol. 2007;133(10):771-782. doi:10.1007/ s00432-007-0222-0

15. Azad AA, Zoubeidi A, Gleave ME, Chi KN. Targeting heat shock proteins in metastatic castration-resistant prostate cancer. Nat Rev Urol. 2015;12(1):26-36. doi:10.1038/nrurol.2014.320

16. Graner M, Raymond A, Romney D, et al. Immunoprotective activities of multiple chaperone proteins isolated from murine B-cell leukemia/lymphoma. Clin Cancer Res. 2000;6(3):909-915.

17. Antognelli C, Gambelunghe A, Muzi G, Talesa VN. Peroxynitritemediated glyoxalase I epigenetic inhibition drives apoptosis in airway epithelial cells exposed to crystalline silica via a novel mechanism involving argpyrimidine-modified Hsp 70, JNK, and NF-кB. Free Radic Biol Med. 2015;84:128-141. doi:10.1016/j.freeradbiomed. 2015.03.026

18. Antognelli C, Gambelunghe A, Muzi G, Talesa VN. Glyoxalase I drives epithelial-to-mesenchymal transition via argpyrimidine-modified Hsp70, miR-21 and SMAD signalling in human bronchial cells BEAS-2B chronically exposed to crystalline silica Min-U-Sil 5: transformation into a neoplastic-like phenotype. Free Radic Biol Med. 2016;92:110-125. doi:10.1016/j.freeradbiomed.2016.01.009

19. Antognelli C, Trapani E, Delle Monache S, et al. KRIT1 loss-offunction induces a chronic Nrf2-mediated adaptive homeostasis that sensitizes cells to oxidative stress: implication for cerebral cavernous malformation disease. Free Radic Biol Med. 2018;115:202-218. doi:10.1016/j.freeradbiomed.2017.11.014

20. Antognelli C, Trapani E, Delle Monache S, et al. Data in support of sustained upregulation of adaptive redox homeostasis mechanisms caused by KRIT1 loss-of-function. Data Brief. 2017;16:929-938. doi:10.1016/j.dib.2017.12.026

21. Rappa F, Sciume C, Lo Bello M, et al. Comparative analysis of Hsp10 and Hsp90 expression in healthy mucosa and adenocarcinoma of the large bowel. Anticancer Res. 2014;34(8):4153-4159.

22. Saini J, Sharma PK. Clinical, prognostic and therapeutic significance of heat shock proteins in cancer. Curr Drug Targets. 2017. doi:10.2174/1389450118666170823121248

23. Fan W, Fan SS, Feng J, et al. Elevated expression of HSP10 protein inhibits apoptosis and associates with poor prognosis of astrocytoma. PLoS One. 2017;12(10):e0185563. doi:10.1371/journal.pone.0185563

24. Chu S, Wen Q, Qing Z, et al. High expression of heat shock protein 10 correlates negatively with estrogen/progesterone receptor status and predicts poor prognosis in invasive ductal breast carcinoma. Hum Pathol. 2017;61:173-180. doi:10.1016/j.humpath.2016.09.039

25. Feng J, Luo J, Wang H, et al. High expression of heat shock protein 10 (Hsp10) is associated with poor prognosis in oral squamous cell carcinoma. Int J Clin Exp Pathol. 2017;10(7):7784-7791. 
26. Feng J, Xie G, Yuting Z, et al. Elevated HSP90 associates with expression of HIF-1 $\alpha$ and p-AKT and predicts the poor prognosis in the nasopharyngeal carcinoma. Histopathology. 2019. doi:10.1111/ his. 13862

27. Xu L, Jiang Y, Zheng J, et al. Aberrant expression of $\beta$-catenin and Ecadherin is correlated with poor prognosis of nasopharyngeal cancer. Hum Pathol. 2013;44(7):1357-1364. doi:10.1016/j.humpath.2012. 10.025

28. Vasuri F, Malvi D, Bonora S, et al. From large to small: the immunohistochemical panel in the diagnosis of early hepatocellular carcinoma. Histopathology. 2018;72(3):414-422. doi:10.1111/ his. 13389

29. Chen J, Gao T, Wan S, Zhang Y, Yang J, Yu Y, Wang W. Genomewide identification, classification and expression analysis of the HSP gene superfamily in tea plant (Camellia sinensis). Int J Mol Sci. 2018;19(9).pii:E2633. doi:10.3390/ijms19092633

30. Rappa F, Farina F, Zummo G, et al. HSP-molecular chaperones in cancer biogenesis and tumor therapy: an overview. Anticancer Res. 2012;32(12):5139-5150

31. Ciocca DR, Calderwood SK. Heat shock proteins in cancer: diagnostic, prognostic, predictive, and treatment implications. Cell Stress Chaperones. 2005;10(2):86-103. doi:10.1379/csc-99r.1

32. Rappa F, Pitruzzella A, Marino Gammazza A, et al. Quantitative patterns of Hsps in tubular adenoma compared with normal and tumor tissues reveal the value of Hsp10 and Hsp60 in early diagnosis of large bowel cancer. Cell Stress Chaperones. 2016;21(5):927-933. doi:10.1007/s12192-016-0721-5

33. Cappello F, David S, Rappa F, et al. The expression of HSP60 and HSP10 in large bowel carcinomas with lymph node metastase. BMC Cancer. 2005;5:139. doi:10.1186/1471-2407-5-64

34. Shan YX, Liu TJ, Su HF, et al. Hsp10 and Hsp60 modulate Bcl-2 family and mitochondria apoptosis signaling induced by doxorubicin in cardiac muscle cells. J Mol Cell Cardiol. 2003;35(9):1135-1143.
35. Murphy ME. The HSP70 family and cancer. Carcinogenesis. 2013;34 (6):1181-1188. doi:10.1093/carcin/bgt111

36. Giri B, Sethi V, Modi S, et al. Heat shock protein 70 in pancreatic diseases: friend or foe. J Surg Oncol. 2017;116(1):114-122. doi:10.1002/jso. 24653

37. Wang Z, Gou W, Liu M, Sang W, Chu H, Zhang W. Expression of P53 and HSP70 in chronic hepatitis, liver cirrhosis, and early and advanced hepatocellular carcinoma tissues and their diagnostic value in hepatocellular carcinoma: an immunohistochemical study. Med Sci Monit. 2015;21:3209-3215. doi:10.12659/msm.8 95592

38. Lee HW, Lee EH, Kim SH, Roh MS, Jung SB, Choi YC. Heat shock protein 70 (HSP70) expression is associated with poor prognosis in intestinal type gastric cancer. Virchows Arch. 2013;463(4):489-495. doi:10.1007/s00428-013-1461-x

39. Gunaldi M, Kocoglu H, Okuturlar Y, et al. Heat shock protein 70 is a useful marker for predicting colorectal cancer. J BUON. 2015;20 (6):1464-1470.

40. Tang T, Yang C, Brown HE, Huang J. Circulating heat shock protein 70 is a novel biomarker for early diagnosis of lung cancer. Dis Markers. 2018;2018:6184162. doi:10.1155/2018/6184162

41. Budina-Kolomets A, Webster MR, Leu JI, et al. HSP70 inhibition limits FAK-dependent invasion and enhances the response to melanoma treatment with BRAF inhibitors. Cancer Res. 2016;76 (9):2720-2730. doi:10.1158/0008-5472.CAN-15-2137

42. Dimas DT, Perlepe CD, Sergentanis TN, et al. The prognostic significance of Hsp70/Hsp90 expression in breast cancer: a systematic review and meta-analysis. Anticancer Res. 2018;38(3):1551-1562. doi:10.21873/anticanres. 12384

43. Wu PS, Chang YH, Pan CC. High expression of heat shock proteins and heat shock factor-1 distinguishes an aggressive subset of clear cell renal cell carcinoma. Histopathology. 2017;71(5):711-718. doi:10.1111/his.13284
Cancer Management and Research

\section{Publish your work in this journal}

Cancer Management and Research is an international, peer-reviewed open access journal focusing on cancer research and the optimal use of preventative and integrated treatment interventions to achieve improved outcomes, enhanced survival and quality of life for the cancer patient.

\section{Dovepress}

The manuscript management system is completely online and includes a very quick and fair peer-review system, which is all easy to use. Visit http://www.dovepress.com/testimonials.php to read real quotes from published authors. 